\title{
Supportive Therapy for Fatigue in Hemodialysis Patients
}

\author{
Ramai $\mathbf{P}^{1}$, Diana Lobo ${ }^{2}$ \\ ${ }^{1}$ Professor, Sri Siddhartha College of Nursing, Tumkur, Karnataka and PhD Scholar, Rajiv Gandhi University of \\ Health Sciences \\ ${ }^{2}$ Professor, Department of Medical Surgical Nursing, Laxmi Memorial College of Nursing, Mangalore, \\ Karnataka
}

Corresponding Author: Ramai P

\begin{abstract}
Fatigue is an enervating symptom of prolonged dialysis of patients and significantly impacts the health related quality of life of dialysis patients. Reduction of fatigue in dialysis patients is a challenging task for any health care provider. Fatigue develops during long term dialysis usually due to chronic health conditions associated with prolonged dialysis. The contributing factors for fatigue in end stage renal disease (ESRD) patients may be broadly classified into physiological, psychological / behavioural, socio-demographic and dialysis related factors. It is known that some of these factors are modifiable leading to reduction in fatigue. A multidisciplinary health care strategy comprising alternative therapy such as acupressure; mind based therapy such as meditation, deep breathing and yoga; body based therapies such as physical activity, therapeutic exercise, body massage; biological based therapy such as diet and nutrition shall help to reduce the fatigue in dialysis population.

Conclusion: To improve the patient care and health related quality of life in dialysis patients, nurses should develop a framework for decreasing the fatigue. This concept paper describes the various therapies available for reducing the fatigue and discusses the ways of including these supportive and alternative therapies into regular medical care.
\end{abstract}

Keywords: Fatigue, ESRD, hemodialysis, alternative and supportive therapy

\section{INTRODUCTION}

A gradual reduction in the overall quality of life of ESRD patients due to comorbid conditions, dietary restrictions, side effect of medications and dialysis is quite common. Severe fatigue is one of the major complaints of most of the ESRD patients who are on dialysis. Fatigue is the subjective sense of weakness, lack of energy and tiredness which is mostly neglected by healthcare workers since there is an established mindset that the dialysis population must learn or adapt to live fatigue. On the contrary, there are many alternative and supportive therapies which may improve fatigue, leading to a better quality of life for the patient. This paper focuses on conceptualizing the alternative and supportive interventions for fatigue management in ESRD patients on dialysis

\section{Contributing factors for fatigue in ESRD patients}

In ESRD patients, the development of fatigue has a complex etiology which may be broadly classified under four categories.

\section{Physiological factors}

Anaemia, malnutrition, uremia, interdialytic weight gain, hyperparathyroidism, adverse effect of medications, pruritus, inflammation, decreased albumin level in blood, physical inactivity, dietary and fluid 
restriction, comorbidities like cancer and congestive cardiac failure

\section{Socio demographic factors}

Age, sex, race, employment status, marital status, education, social support, social functioning

\section{Psychological and behavioral factors}

Anxiety, cognitions, stress, depression, sleep disorders, substance use, mood disorders.

\section{Dialysis related factors}

Post dialysis fatigue, dialysis adequacy, mode of dialysis, frequency and duration of dialysis 1

\section{Pathogenesis}

Pro-inflammatory cytokines are the potential mediators of fatigue. The cytokines provide a common biological pathway for physiological, psychological and dialysis related factors leading to development of fatigue during dialysis (Figure 1). Biochemical markers like decreased albumin and uremia leads to PEM, nausea and loss of appetite which in turn trigger fatigue in dialysis patients. ESRD patients experience constant lack of energy due to physical fatigue which is worst on dialysis days. This leads to further decline in mental and physical activity, role limitation, decreased ability to concentrate on conversation and reduced ability to remember. ${ }^{[1]}$

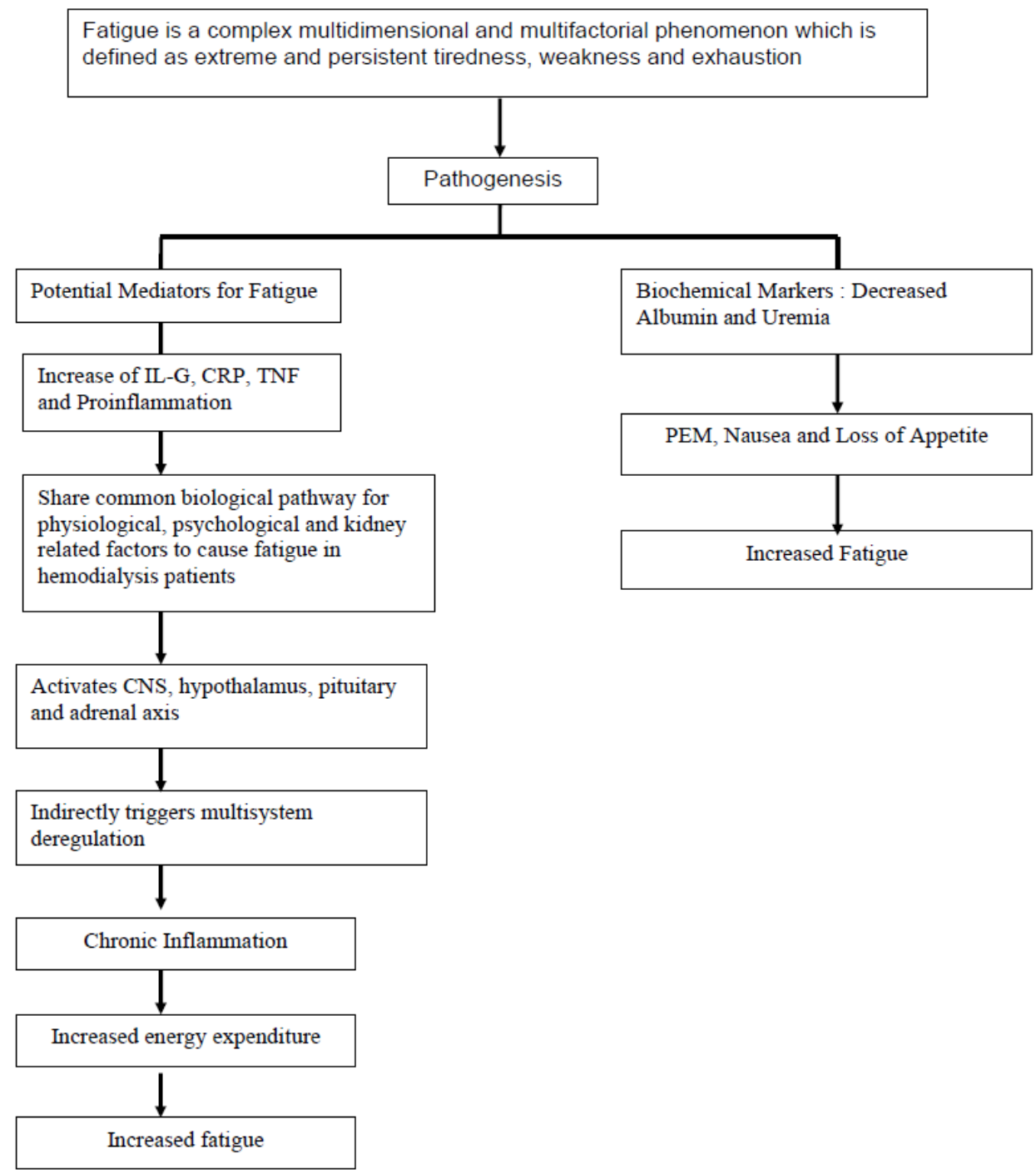

Figure 1 Etiopathogenesis of Fatigue in Patient Population Undergoing Hemodialysis 


\section{Symptoms of fatigue}

Fatigue can cause physical, mental and emotional symptoms which include: Chronic tiredness or sleepiness, headache, dizziness, sore or aching muscles, muscle weakness, slow reflex and responses, impaired decision making and judgment, moodiness such as irritability, loss of appetite, reduced immune function, short term memory problems, poor concentration and low motivation. ${ }^{[1]}$

\section{Supportive therapy for fatigue}

To improve fatigue, a multidisciplinary approach and its integration into practice is essential. This shall happen with proper co-ordination amongst dialysis technicians, renal dieticians, nephrologists, nephrology nurse, physical therapist and AYUSH specialists. This team must aim not only to clean the blood and prolong the life but also to maintain the quality of life of renal patients (Figure 2)

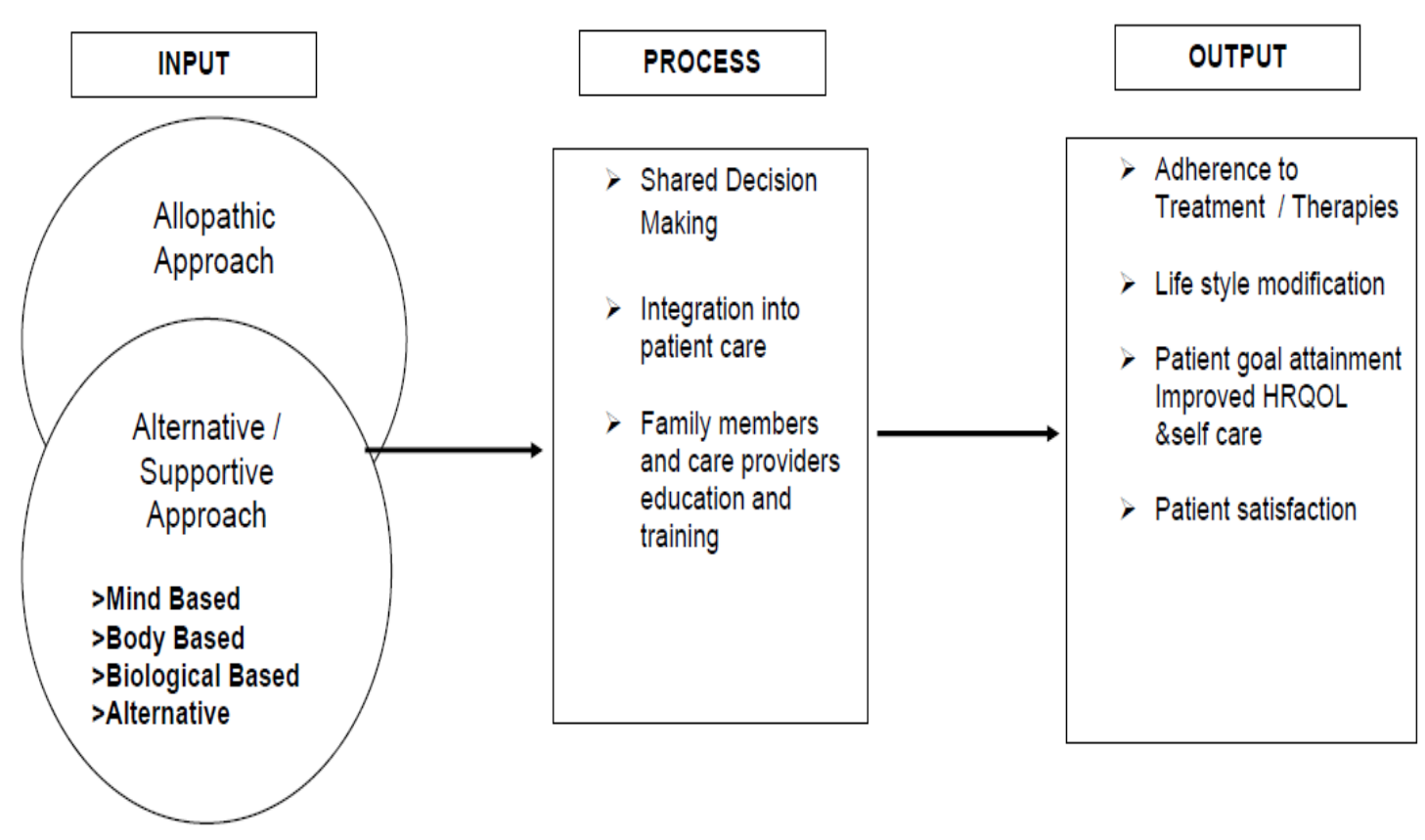

Figure 2 Conceptual Framework on Supportive Therapy for Fatigue in Hemodialysis Population

\section{Allopathic Interventions}

Some of the pharmacological approaches for fatigue management in renal patients are: dialysis, administration of erythropoietin, anti-depressants, anxiolytics, levocartinine and human growth hormones. [1]

\section{Alternative / Supportive Interventions}

Mind based therapy - Meditation, deep breathing and Yoga

Body based therapy - Physical activity, therapeutic exercise, body massage

Biological based therapy - Diet and nutrition
Alternative therapy - Accupressure

\section{Mind Based Therapy Yoga}

Yoga involves aspects such as following specific diet pattern, appropriate mental attitude and practicing pranayamas (breathing exercise) and asanas (posture) as well as meditation to attain the highest level of consciousness. Yogic breathing exercises lead to stretching of lungs resulting in production of inhibitory signals from cardiorespiratory region. These signals are believed to induce changes in autonomic nervous system by synchronization of 
neural elements resulting in a condition of reduced metabolism and parasympathetic dominance.

It has been reported that 30 minutes of hath yoga practice daily for 4 months showed a significant reduction in oxidative stress (malondialdehyde, protein oxidation, and phospholipase A2 activity) and increase in antioxidant activity (superoxide dismutase and catalase activities) in patients with CKD who were on hemodialysis. ${ }^{[2,3]}$ Yoga reduces sympathetic tone and improves parasympathetic tone. There are studies showing that yoga reduces sympathetic tone and improves parasympathetic tone and the significant role played by yoga in controlling renal dysfunction as assessed by blood urea and serum creatinine values. The patients undergoing the yogic exercise regime along with conventional treatment showed a significant reduction in blood urea and serum creatinine value over a period of 6 months. ${ }^{[2]}$

Benefits of yoga include increased muscular strength, flexibility, range of motion, relaxation and a sense of wellbeing. Yoga also helps in decreasing pain, improving fatigue and sleep quality, stress reduction and control over physiological parameters. ${ }^{[4]}$

\section{Deep breathing relaxation techniques}

These techniques stimulate the parasympathetic nervous system thereby increasing endorphin production, decreasing heart rate and increasing lung expansion. This leads to improvement in removal of toxins and metabolic wastes and production of more energy due to increased metabolism. Deep breathing exercises maximize the amount of oxygen supplied to the tissues resulting in production of more energy leading to reduction in levels of fatigue. Reduction in oxidative stress, increase of cellular energy and elasticity of blood vessels and improved circulation are the benefits of deep breathing relaxation techniques. ${ }^{[5]}$

\section{Body Based Therapy \\ Massage}

Massage involves the palpation of soft tissue and muscles and is a simple and non-invasive method for ensuring relaxation. Massage leads to both physical and mental relaxation and is capable of generating energy transmission between the practitioner and the subject which in turn improves fatigue in HD patients leading to improvement in quality of life. ${ }^{[6]}$

\section{Regular Exercise}

Regular exercise program for hemodialysis patients results in strengthening of muscles, improvement in flexibility of joints, reduction in musculoskeletal pain, increased appetite, improvement in quality of sleep and reducing fatigue. ${ }^{[7]}$ During exercise, blood vessels in active skeletal muscles vasodilate. Autonomic activation of the exercise pressor reflex occurs in part due to the stimulation of thin fibre in muscle afferents whose free nerve endings are located in the interstitium of contracting skeletal muscle. [8] Intradialytic exercise improves urea clearance by opening vascular beds in the working muscle and exposing more tissue which allows the fluid in the tissue to move to the intravascular compartment. Potassium and phosphate ions shifting from the intracellular compartment into muscle interstitial fluid occurs during exercise. ${ }^{[9]}$

It has been reported that high physical activity was associated with favourable results for most health-related quality of life scale scores, including frailty, disability, and exhaustion in Korean dialysis patients. ${ }^{[10]}$ In another pilot study, exercise induced positive biochemical modifications in muscles in CKD patients were reported. [11] One more article reviewed the positive effects of different intradialytic exercise training on physical functions and health related QOL of hemodialysis patients. ${ }^{[12]}$ Another study reported that cognitive decline in psychomotor speed and executive function is possibly prevented by intradialytic cognitive training and exercise 
training. ${ }^{[13]}$ It has been also shown that exercise during hemodialysis could increase muscle capacity and would be helpful for maintaining the albumin level in hemodialysis patients. ${ }^{[14]}$

A review article reported that factors contributing to sustainable exercise programs include: encouragement to exercise intradialytically; dialysis and medical staff commitment; adequate physical requirements of equipment and space. It has been reported that there is no age barrier for patients to exercise during hemodialysis. ${ }^{[15]}$

\section{Biological Based Therapy Diet and Nutrition}

Intake of adequate protein and calories, safe levels of sodium, potassium, phosphorous and fluids are important for dialysis patients. Tailored nutritional intervention based on restrictions for the patients may result in improved albumin levels even among patients with high $\mathrm{c}$ reactive proteins. A nurse led intervention educating the patients on hyperphoshetemia, its symptoms; use of phosphate binder with their benefits for improving health related quality of life will improve albumin levels and reduce hyphosphetemia. Creating consistent interactive communication, emphasizing positive action, typical food pattern, focusing on a single goal instead of multiple goals can promote adherence to nutritional advice. ${ }^{[16]}$

\section{Alternative therapies Acupressure}

Acupressure medicine is considered as a clinical and comprehensive nursing intervention and is widely used. Acupressure medicine, as a healing art and manual treatment is beautifully integrated with nursing. From psychological aspect, acupressure is reported to induce alpha brain wave stimulation that consequently leads to relaxation and reduction of fatigue and anxiety. Acupressure has a positive effect on the body and mind through stimulation of opioid - endogen systems such as endorphin and encephalin leading to a happy mental state. ${ }^{[17]}$

\section{Shared Decision Making (SDM)}

Shared decision making between patients and providers are ethical and can preserve patient autonomy, promote truthfulness and openness, consider patient values and preferences and may lead to improved health outcome. SDM helps in implementation of integrative approach into patient care and is associated with improved health outcomes across a range of illness. [18]

\section{Integrative approach}

Open communication between patients, family members and health care providers of both allopathic and supportive/alternative therapies is essential. If all three parties are actively involved in treatment decisions, it decreases decisional conflict and manage uncertainty. Integrative approach nurtures a partnership that maximises patient centered outcomes. ${ }^{[18]}$

\section{Family Member and Care Provider Education}

For integrative approach to be successful, care providers and family members require training and education on alternative and supportive treatments as well as referral processes for integrating the modalities into the care of their patients. The education process should begin at the early stage of disease and continue as an ongoing learning process. The risks and benefits of all possible therapeutic options must be considered in addition to considering patient values, beliefs and preferences. ${ }^{[18]}$

The practice of integrative healthcare with shared decision-making may lead to improved preventive and health promotion behaviour, adherence to treatment, lifestyle modification, patient satisfaction, goal setting and attainment .Ultimately patients get motivated to make behavioural changes which can improve health related quality of life and self care. 


\section{Patient satisfaction}

Patients perceive their own health as better when they are incorporated into an integrative health plan that aligns with their personal values

\section{Patient goal attainment}

Patients whose providers encourage them to set their own goals are more likely to achieve their goals. This is embedded in the concept of integrative approach and shared decision making

Practical suggestions for providers to start the integrative approach are:

A. Identifying the difficulties patients may have in understanding treatment options

B. Understanding about the difficulties of the patients in adhering to the previous or current prescribed treatment

C. Soliciting information on patient values and general preferences of care

D. Asking about patients current or past use of alternative therapy

Allopathic approach combined with patient specific supportive therapy can provide biobehavioral integrative approach to fatigue management. Such approach takes into account patients preferences and beliefs with the support of their healthcare provider

\section{CONCLUSION}

Allopathic treatment approach with alternative / supportive therapy for fatigue management in hemodialysis patients remains a challenge. All parties have an important role and a shared responsibility to work towards patient-centered care. Improved patient communication using an input - process- output framework may lead to increased understanding of disease etiology as well as better interdisciplinary approach to health care delivery. In case of individual care planning, an innovative and holistic treatment pattern is possible since patients and health care providers actively participate in the care planning. As a result of shared responsibility, patients tend to sustain health behaviour changes and make better use of healthcare services.

\section{Acknowledgement: None}

\section{Conflict of Interest: None}

\section{Source of Funding: None}

\section{REFERENCES}

1. Manisha J, Steven DW, Jennifer LS et al. Fatigue in patients receiving maintenance dialysis: A review of definitions, measures and contributing factors.Am J Kidney Dis. 2008: 52 (2): 353-365.

2. Pandey RK, Arya TV, Kumar A et al. Effects of 6 months yoga program on renal functions and quality of life in patients suffering from chronic kidney disease. Int $\mathbf{J}$ Yoga. 2017; 10 (1): 3-8.

3. Lorenzo G, Donvan AM, Yeiny TP et al. Effect of yoga therapy on oxidative stress indicators with end stage renal disease on hemodialysis. Int J Yoga. 2013; 6(1): 31-38.

4. Katja B, Thomas O, Stefania $M$ et al. Effects of yoga interventions on fatigue: a meta analysis: Evidence-Based Complementary and Alternative Medicine. 2012: article ID 124703; 9 pages.

5. Sutinah, Rasyidah A. The effects of relaxation breathing on fatigue in patients with chronic kidney disease undergoing hemodialysis. Malahayati Int J Nur Health Sci. 2020: 3(1);15-21.

6. Kevser SU, Reva BA. The effect of foot reflexology and back massage on hemodialysis patients' fatigue and sleep quality. Complimentary Therapy in Clin Prac. 2016: 24; 139-144.

7. Jahangir R, Alireza A, Mansour R et al. Effect of regular exercise program on depression in hemodialysispatients. Int Scholarly Res Notices. 2015: article ID 182030.

8. Lott MEJ, Hogeman CS, Vickery L et al. Effects of dynamic exercise on mean blood velocity and muscle interstitial metabolite responses in humans. Am J Physiol Heart Circ Physiol. 2001: 281; H1734-H1741.

9. Paluchamy $T$, Vaidhyanathan $R$. Effectiveness of Intradialytic exercise on dialysis adequacy, physiological parameters, biochemical markers and quality of life - A Pilot Study. Saudi J Kidney Dis Transpl. 2018 : 29(4); 902-910.

10. Kang SH, Do JY, Jeong HY et al. The Clinical Significance of Physical Activity in 
Maintenance of Dialysis Patients. Kidney Blood Press Res. 2017: 42;575-586.

11. Bullani R, El-Housseini Y, Giordano F et al. Effect of intradialytic resistance band exercise on physical function in patients on Maintenance hemodialysis:A Pilot Study. J Renal Nutrition. 2011: 21(1); 61-65.

12. Gomes Neto M, de Lacerda FFR,Lopes AA et al. Intradialytic exercise training modalities on physical functioning and health-related quality of life in patients undergoing maintenance hemodialysis: systematic review and meta-analysis. Clinical Rehabilitation. 2018: 32(9).

13. McAdams-DeMarco MA, Konel J, Warsame $\mathrm{F}$ et al. Intradialytic Cognitive and Exercise Training May Preserve Cognitive Function. Kidney Int Rep. 2018: 3; 81-88.

14. Tayebi M, Ramezani A, Kashef M. The effect of intradialytic isometric resistance training on muscle capacity and serum albumin levels in hemodialysis patients. Nephro-Urol. 2018: 10; (2:e65081)
15. Bennett PN, Breugelmans L, Barnard R, Agius M, Chan D, Fraser D et al. Sustaining a hemodialysis exercise program: A review. Seminars in Dialysis. 2010: 23(1); 62-73.

16. Bryal D, Ravindra $P$, Bhaskaran $U$ et al. Effect of multidimensional educational interventions among dialysis patients. The Open Urology and Nephrology Journal. 2018: 11; 72-78.

17. Fakhri S, Leila K, Mahboubeh V et al. Effect of acupressure on fatigue in patients on hemodialysis. Iran J Nurs Midwifery Res. 2013: 18(6); 429-434.

18. Allysa TB, Leanne S, Gwenyth RW. Shared decision making: A fundamental tenant in a conceptual framework of integrative healthcare delivery. Integrative Medicine Insights. 2013: 8; 29-36S.

How to cite this article: Ramai P, Lobo D. Supportive therapy for fatigue in hemodialysis patients. Int J Health Sci Res. 2021; 11(7): 367-373. DOI: https://doi.org/ 10.52403/ijhsr. 20210750 